\title{
Technology Using Neural Network Optimization Path in Packet Switching Network
}

\author{
Shoubai Xiao \\ NanChang Institute of Science \& Technology
}

Keywords: Dynamic network, Neural network, Genetic algorithm, The shortest path

\begin{abstract}
How to find the optimal path between two nodes has been one of the most difficult problems in the internet. In this paper, a method of finding the optimal path based on neural network technology is proposed, which solves the problem of finding the optimal path by adjusting the weight of the neuron. The results show that the algorithm proposed in this paper is simple in calculation and fast in convergence speed, and it is suitable for the research on the optimal path of packet switching. Although the shortest path algorithm has been established, the researchers are still studying other better path selection methods.
\end{abstract}

\section{Introduction}

Modern communication network is widely used in TCP/IP TCP/IP network architecture, network architecture, network layer is very important to the network level, the main function is the Internet layer packet (data unit Internet layer) routing and forwarding packets, this process is called routing. Routing is the most important function of the Internet, especially in packet switched networks. Routing technology has a great impact on the performance of the network. The most ideal routing algorithm is to find the shortest path for the source node and the target node, and to transmit data at high speed. However, to find the shortest path between two nodes is a well-known problem.

In a packet-switched network, packet communication between two hosts is generally done by sending the host to organize the data into blocks of data, commonly referred to as packets, in which the network address of the destination host (generally called an IP address). The routing device in the network according to the packet carrying the destination address for the packet to find the path and forward, and ultimately reach the target host. The main goal of a routing policy is to minimize the propagation delay of IP packets and to transmit packets as far as possible. The main factors that affect the average packet transmission delay time are the reliability of the network, the capacity of the network bandwidth and the network routing. The effect of the route on the network performance is very important. Therefore, an ideal routing algorithm should try to find the optimal path within the specified time to meet the network QOS.

\section{Introduction to Basic Genetic Algorithm}

Genetic algorithm is similar to natural evolution, by acting on the chromosome to find a good chromosome to solve the problem. Similar to nature, genetic algorithms have no knowledge of the problem itself. All that is needed is to evaluate each chromosome produced by the algorithm and to select the chromosomes based on the fitness value, so that the more adaptive chromosomes have more Of the breeding opportunities. In the genetic algorithm, a number of problems, such as chromosomes, are generated in a random way. The initial population is formed. Each individual is evaluated by the fitness function, and the individuals with low fitness are selected. Participate in the genetic operation, after genetic manipulation of individual collections to form the next generation of new populations, the new population of the next round of evolution.

It is generally believed that genetic algorithm has five basic components (which is summarized by Michalewicz):

(1) Genetic Representation of Solution to Problem

(2) A Method for Creating Initial Populations of Solutions

(3) According to individual fitness value to judge the merits of the evaluation function 
(4)Genetic operator produced by individual genetics in the original process of changing replication

(5) Parameters of genetic algorithm

The genetic algorithm maintains a population $\mathrm{P}(\mathrm{t})$ consisting of a population of individuals $(\mathrm{t}$ represents genetic algebra). Each body represents a potential solution to the problem. Each individual is evaluated for fitness and gets its fitness. Some individuals undergo a random transformation called a genetic operation, thereby generating new individuals. There are two main methods of transformation: mutation (mutation) method is to change an individual to produce new individuals; hybrid (crossover) method is to combine the relevant parts of the two individuals to form a new individual. The newly generated individuals (called offspring) $\mathrm{C}$ ( $\mathrm{t}$ ) continue to be evaluated for merit. A new population is formed by selecting the superior individuals from the parental and progeny populations. After several generations, the algorithm converges to an optimal individual, which is likely to represent the optimal or suboptimal solution of the problem.

General structure of the genetic algorithm is described as below:

Begin

$\mathrm{t} \leftarrow 0$

Initialize $\mathrm{P}(\mathrm{t})$

Evaluate $\mathrm{P}(\mathrm{t})$

While (Termination conditions are not met) do

Restructure $\mathrm{P}(\mathrm{t})$ to produce $\mathrm{C}(\mathrm{t})$

Evaluate $\mathrm{C}(\mathrm{t})$

Choose $\mathrm{P}(\mathrm{t}+1)$ from $\mathrm{P}(\mathrm{t})$ and $\mathrm{C}(\mathrm{t})$

$\mathrm{t} \leftarrow \mathrm{t}+1$

end

end

\section{Introduction to Basic Neural Network}

Neural network is composed of a large number of simple and non-linear processing unit (neurons, processing components, electronic components, optoelectronic components, etc.) reflecting the essential characteristics, which are widely connected to form a complex network system. It is based on modern neuroscience research, reflecting the basic characteristics of human brain function. But it is not a true portrayal of the human brain system, but only to some of its simplification, abstraction and simulation. Neural network is a highly nonlinear continuous super-large-scale dynamic system, which is characterized by the distribution of information storage and information in parallel co-processing. Although the structure of a single neuron is extremely simple, limited function, but a large number of neurons constitute the network system to achieve the behavior is rich and colorful. Neural network parallel processing capacity is reflected by the distributed structure, that is, by a different number of neurons and their connection between the different forms and methods to represent the process. The operation of neural network is the transfer process of the value from input to output, and the information is stored and calculated at the same time when the value is transmitted, so that the access and calculation of information are well integrated.

The reason why the brain can handle extremely complex analysis, reasoning, on the one hand because of its large number of neurons, on the other hand is that the neuron can input signal nonlinear processing. As a result, a nearer neuron model, which is a nonlinear unit of multi-input single-output, can be established. Where $\mathrm{F}(\mathrm{x})$ is the non-linear transfer function of the connection strength between the neurons. 


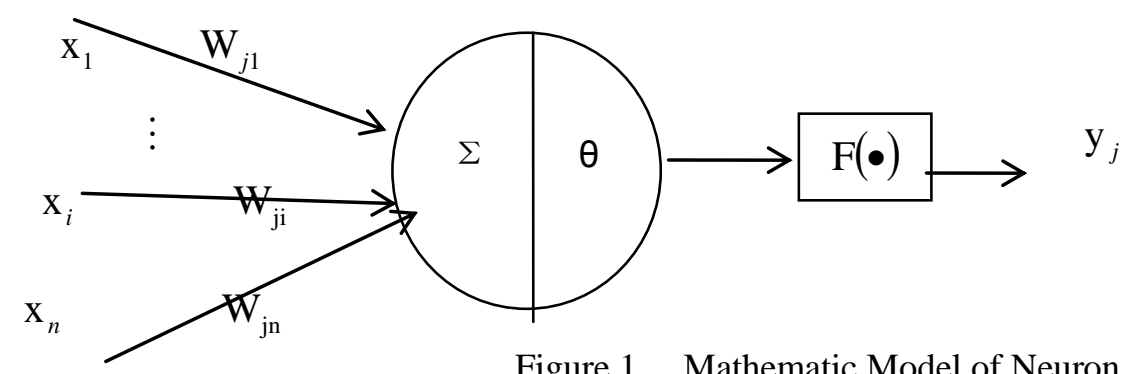

\section{Multilayer Feedforward Network}

Multi-level network, as the name implies, consists of multiple functional layers of the network, this structure of the network, in addition to the data output layer and data input layer accidents, including hidden layer (or hidden unit), each level. Multi-layer feedforward neural network is a typical hierarchical structure in neural network. The information of input layer neurons goes from the input layer to the hidden layer neuron network and then forwards to the output layer, and between the neurons and the neurons. The weight of the connection is called the link weight. Modern networks are generally hierarchical structure of the network, the most famous of which are ISO seven-layer architecture and the actual use of Internet TCP / IP architecture, the network architecture is hierarchical, are multi-level network structure. The network nodes in the communication network correspond to the neurons of the neural network. There are link links between the nodes and the nodes. Each link has the corresponding link weights. The link links between the neuron node and the output node also have links Weight value, the relationship between the two shown in Fig. 2.
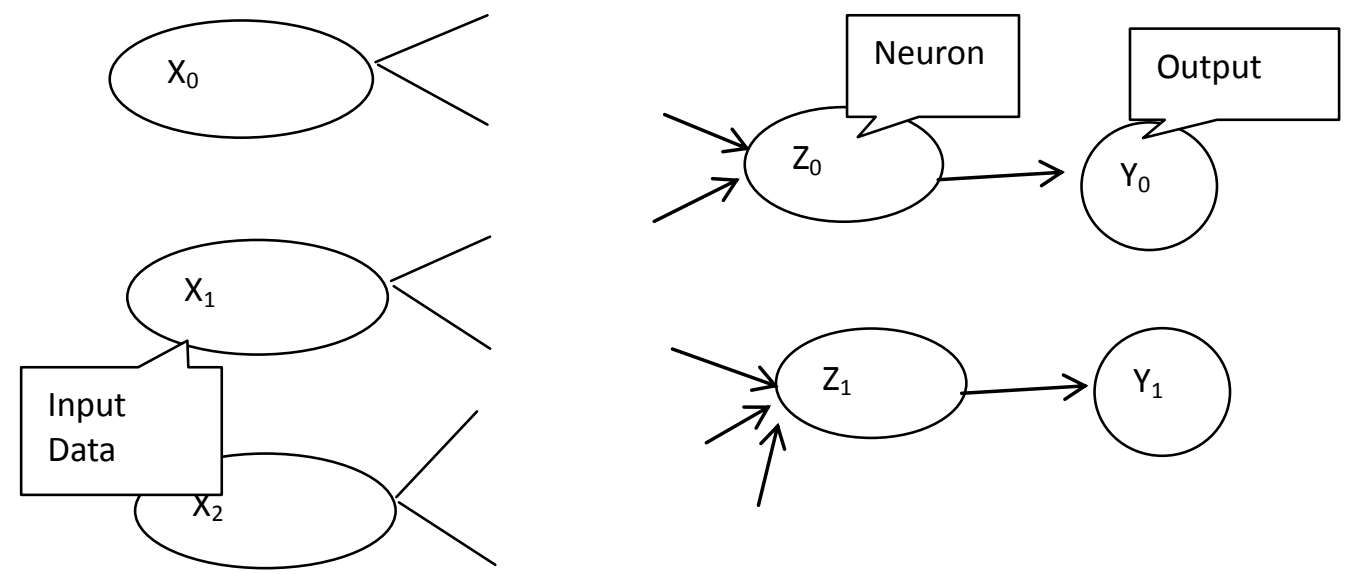

Figure 2. Diagram of Multilayer Feedforward Network

\section{Conclusion}

Neural network has been proved to be a simple method to optimize packet-switched multi-layer network, and the network scale gradually increases, which makes it difficult to find the shortest path between network nodes. In practical environment, because of the dynamic nature of the network, it is very important to maintain the shortest path between nodes. When the topological structure of the network changes, if the traditional static shortest path algorithm to re-solve the shortest path between network nodes, the system load will be greatly increased. In terms of the amount of calculation or time consuming, it is difficult to accept. Therefore, it is very important to seek a new algorithm for shortest path updating in dynamic environment. In order to update the dynamic shortest path algorithm (ball line model), this paper improves its update operation, and increases the operation module that the nodes are dropped from the network and the nodes join the network. From the experimental analysis, it is found that the improved algorithm improves the performance of the ball line model.

Project Supported by. Nanchang Key Laboratory of Intelligent Building Network Engineering 


\section{References}

[1] Galand C, Lebizay G, Menez J, et al. System for coding voice signals to optimize bandwidth occupation in high speed packet switching networks: US, US 6104998 A [P]. 2000.

[2] Prakash P S, Selvan S. An Efficient and Optimized Multi Constrained Path Computation for Real Time Interactive Applications in Packet Switched Networks [J]. International Journal of Computer Science, 2008(4).

[3] Che X, Hunter D K, Henning I D. Buffer-Optimized Traffic Smoothing for Optical Packet Switching Access Network[C]// International Conference on Transparent Optical Networks. IEEE, 2006:6-9.

[4] Zaleski A, Kacprzak T. Packet Switching Networks Tra ffi c Prediction Based on Radial Basis Function Neural Network [J]. Technical University of Lódź Institute of Computer Science, 2010.

[5] Che X, Hunter D K, Henning I D. Buffer-Optimized Traffic Smoothing for Optical Packet Switching Access Network[C]// International Conference on Transparent Optical Networks. IEEE, 2006:6-9.

[6] Salas E H. Design of Optical Buffer Architectures for Packet-Switched Networks [J]. Lap Lambert Academic Publishing, 2010.

[7] Chaudhary A, Pandey N K. Genetic algorithm for shortest path in packet switching networks[J]. Journal of Theoretical \& Applied Information Technology, 2011.

[8] Ko H, Lee G, Pack S. Optimized and distributed data packet forwarding in LTE/LTE-A networks[C]// IEEE International Conference on Communications. IEEE, 2014:2502-2507.

[9] Durand F R, Abrao T. Energy-efficient power allocation for WDM/OCDM networks with particle swarm optimization [J]. Journal of Optical Communications \& Networking, 2013, 5(5):512-523.

[10]Henderson T, Bargallo J, Beppler D R, et al. Centralized femtocell optimization: US, US9210583 [P]. 2015.

[11] White, Patrick E. Multi-access terminal with capability for simultaneous connectivity to multiple communication channels [J]. 2009.

[12]Pizzo M J, Sundaresan S, Pamulapati R P, et al. Systems and methods for requesting and receiving database change notifications: US, EP1462958[P]. 2004. 